\title{
Properties of right fractional sum and right fractional difference operators and application
}

\section{Zuoshi Xie ${ }^{1}$ and Chengmin Hou ${ }^{2 *}$}

\section{"Correspondence:}

cmhou@foxmail.com

${ }^{2}$ Department of Mathematics,

Yanbian University, Yanji, 133002,

P.R. China

Full list of author information is

available at the end of the article

\section{照 Springer}

\begin{abstract}
In this paper, the concepts of a right fractional sum and right fractional difference operators are introduced. Some basic properties of a right fractional sum and right fractional difference operators are proved. According to these properties of a right fractional sum and right fractional difference operators, we studied an initial problem and a boundary value problem with two-point boundary conditions. We hope that the present work will facilitate solving a fractional difference equation with right fractional difference operators.
\end{abstract}

MSC: 39A99; 39A12; 26A33

Keywords: right fractional sum; right fractional difference operator; initial problem; boundary value problem

\section{Introduction}

Recently, there appeared a number of papers on the discrete fractional calculus, which has helped to build up some of the basic theory of this area. For example, Atici and Eloe discussed the properties of the generalized falling function, a corresponding power rule for fractional delta-operators, and the commutativity of fractional sums in [1]. Goodrich studied a fractional boundary value problem in [2], which gave the existence results for a certain two-point boundary value problem of right-focal type for a fractional difference equation. The authors of [3] have developed a well-posed initial value problem and proposed multiple solution algorithms. An interesting recent paper by Atici and Sengül [4] addressed the use of fractional difference equations in tumor growth modeling. For recent studies in discrete fractional calculus involving initial boundary value problems, see [5-32].

From the above works, we can see in fact, although the discrete fractional calculus have been studied by many authors, to the best of our knowledge, that the properties of a right fractional difference operator have not been discussed. Our objective is twofold. On one hand we proceed to develop the theory of fractional difference calculus, namely we introduce the concepts of a right fractional sum and right fractional difference operators and prove some basic properties of a right fractional sum and right fractional difference operators. On the other hand, according to these properties of a right fractional sum and right

(c) 2015 Xie and Hou. This article is distributed under the terms of the Creative Commons Attribution 4.0 International License (http://creativecommons.org/licenses/by/4.0/), which permits unrestricted use, distribution, and reproduction in any medium, provided you give appropriate credit to the original author(s) and the source, provide a link to the Creative Commons license, and indicate if changes were made. 
fractional difference operators, we studied an initial problem and a boundary value problem with two-point boundary conditions. The proofs are similar to those of earlier work by both Goodrich [2] and Holm [10]; there nonetheless is something new and interesting here. The contributions of this article aim to initiate the study of right fractional difference operator. This interest is in part due to the useful applications of the fractional calculus together with its interesting and often nontrivial mathematical theory. On the other hand, it might be of interest to see what happens in the case of more complicated boundary conditions or a higher-order problem. Due to the lack of commutativity of the right fractional difference, the sequential boundary value problem is of interest. Furthermore, combining the results of Goodrich [2] and Holm [10], there seem to be considerable possibilities for future work to address the sequential boundary value problems with left and right fractional difference operators, and such investigations might provide interesting future work. We believe that the present work facilitates solving a fractional difference equation with a right fractional difference operator.

\section{Right fractional sum and right fractional difference operators}

In this section, the concepts of a right fractional sum and right fractional difference operators are introduced and their some basic properties are proved.

Denote ${ }_{b} \mathbb{N}:=\{b\}-\mathbb{N}_{0}=\{\ldots, b-2, b-1, b\}, b \in \mathbb{R}$.

Definition 1 (see [2]) We define $t^{\underline{\nu}}:=\frac{\Gamma(t+1)}{\Gamma(t+1-v)}$, for any $t$ and $v$ for which the right-hand side is defined. We also appeal to the convention that if $t+1-v$ is a pole of the Gamma function and if $t+1$ is not a pole, then $t^{\underline{v}}=0$.

Definition 2 The $v$ th order right fractional sum of a function $f$ defined on ${ }_{b} \mathbb{N}$, for $v>0$, is defined to be

$$
{ }_{b} \nabla^{-v} f(t)=\frac{1}{\Gamma(v)} \sum_{s=t+v}^{b}(s-t-1)^{\frac{\nu-1}{}} f(s), \quad t \in_{b-v} \mathbb{N} .
$$

We also define the trivial right sum by ${ }_{b} \nabla^{-0} f(t)=f(t)$, for $t \in{ }_{b} \mathbb{N}$.

Definition 3 Let $f:{ }_{b} \mathbb{N} \rightarrow \mathbb{R}$ and $v>0$ be given, and let $N \in \mathbb{N}$ be chosen such that $N-1<$ $v \leq N$. The $v$ th order right fractional difference of $f$ is given by

$$
\left({ }_{b} \nabla^{v} f\right)(t)={ }_{b} \nabla^{v} f(t)=(-1)^{N} \nabla^{N}{ }_{b} \nabla^{-(N-v)} f(t), \quad t \in{ }_{b-N+v} \mathbb{N} .
$$

Using Definition 3 together with a function $f:{ }_{b} \mathbb{N} \rightarrow \mathbb{R}$ and an order $v>0$ with $N-1<$ $v \leq N$, we may calculate the domain of the $v$ th order right fractional difference as

$$
D\left\{{ }_{b} \nabla^{v} f\right\}=D\left\{\nabla^{N}{ }_{b} \nabla^{-(N-v)} f\right\}=D\left\{{ }_{b} \nabla^{-(N-v)} f\right\}={ }_{b-N+v} \mathbb{N} .
$$

Moreover, the domains of all four sum and difference compositions are given below.

Let $f:{ }_{b} \mathbb{N} \rightarrow \mathbb{R}$ and $v, \mu>0$ be given. Let $N, M \in \mathbb{N}_{0}$ be chosen so that $N-1<v \leq N$ and $M-1<\mu \leq M$. Then

$$
\begin{aligned}
& D\left\{{ }_{b-\mu} \nabla^{-v}{ }_{b} \nabla^{-\mu} f\right\}={ }_{b-\mu-\nu} \mathbb{N}, \quad D\left\{{ }_{b-\mu} \nabla^{v}{ }_{b} \nabla^{-\mu} f\right\}={ }_{b-\mu-N+\nu} \mathbb{N},
\end{aligned}
$$

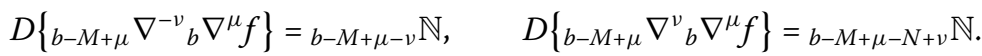


Theorem 1 Let $f:{ }_{b} \mathbb{N} \rightarrow \mathbb{R}$ and $v>0$ be given, with $N-1<v \leq N$. The following two definitions for the right fractional difference ${ }_{b} \nabla^{v} f:{ }_{b-N+v} \mathbb{N} \rightarrow \mathbb{R}$ are equivalent:

$$
\begin{aligned}
& { }_{b} \nabla^{v} f(t)=(-1)^{N} \nabla_{b}^{N} \nabla^{-(N-v)} f(t), \\
& { }_{b} \nabla^{v} f(t)= \begin{cases}\frac{1}{\Gamma(-v)} \sum_{s=t-v}^{b}(s-t-1)^{-v-1} f(s), & N-1<v \leq N, \\
(-1)^{N} \nabla^{N} f(t), & v=N .\end{cases}
\end{aligned}
$$

Proof Let $f$ and $v$ be given as in the statement of the theorem. We are proposing two definitions (2.3) and (2.4) and demonstrating that they are identically equal.

If $v=N$, then (2.3) and (2.4) are clearly equivalent, since in this case,

$$
{ }_{b} \nabla^{v} f(t)=(-1)^{N} \nabla_{b}^{N} \nabla^{-(N-v)} f(t)=(-1)^{N} \nabla^{N}{ }_{b} \nabla^{-0} f(t)=(-1)^{N} \nabla^{N} f(t) .
$$

If $N-1<v<N$, then a direct application of (2.3) yields

$$
\begin{aligned}
& { }_{b} \nabla^{v} f(t)=(-1)^{N} \nabla^{N}{ }_{b} \nabla^{-(N-v)} f(t) \\
& =(-1)^{N} \nabla^{N}\left(\frac{1}{\Gamma(N-v)} \sum_{s=t+N-v}^{b}(s-t-1)^{\frac{N-v-1}{} f(s)}\right) \\
& =(-1)^{N} \nabla^{N-1} \nabla\left(\frac{1}{\Gamma(N-v)} \sum_{s=t+N-v}^{b}(s-t-1)^{\frac{N-v-1}{f}} f(s)\right) \\
& =(-1)^{N} \nabla^{N-1}\left(\frac{1}{\Gamma(N-v)} \sum_{s=t+N-v}^{b}(s-t-1)^{N-v-1} f(s)\right. \\
& -\frac{1}{\Gamma(N-v)} \sum_{s=t+N-v-1}^{b}(s-t)^{\left.\frac{N-v-1}{-} f(s)\right)} \\
& =(-1)^{N-1} \nabla^{N-1}\left(\frac{1}{\Gamma(N-v-1)} \sum_{s=t+N-\nu-1}^{b}(s-t-1)^{\frac{N-\nu-2}{} f(s)}\right) \\
& \vdots \\
& =\frac{1}{\Gamma(-v)} \sum_{s=t-v}^{b}(s-t-1)^{\frac{-v-1}{f}} f(s) .
\end{aligned}
$$

Lemma 1 Let $b \in \mathbb{R}$ and $\mu>0$ be given. Then

$$
\nabla(b-t)^{\underline{\mu}}=-\mu(b-t) \underline{\mu-1}
$$

for any $t$, for which both sides are well defined.

Furthermore, for $v>0$ with $N-1<v \leq N$,

$$
{ }_{b-\mu} \nabla^{-v}(b-t)^{\underline{\mu}}=\mu^{-\underline{v}}(b-t) \stackrel{\mu+v}{\underline{v}}, \quad t \in \in_{b-\mu-\nu} \mathbb{N}
$$

and

$$
{ }_{b-\mu} \nabla^{v}(b-t)^{\mu}=\mu^{\underline{v}}(b-t)^{\mu-v}, \quad t \in \in_{b-\mu-N+v} \mathbb{N} .
$$


Proof It is easy to show (2.5) using the definition of the nabla difference and properties of the gamma function. For (2.6) and (2.7), we first note that $(b-t)^{\mu},(b-t) \frac{\mu+\nu}{}$, and $(b-t) \frac{\mu-v}{\mu}$ are all well defined and positive on their respective domains ${ }_{b-\mu} \mathbb{N},_{b-\mu-\nu} \mathbb{N},_{b-\mu-N+\nu} \mathbb{N}$.

We next show that (2.6) and (2.7) hold. The course of this proof as regards the techniques heavily relies on Holm [10]. For convenience of the reader, we display it as follows.

For $v=1$, we see from direct calculation that

$$
\begin{aligned}
& { }_{b-\mu} \nabla^{-1}(b-t)^{\mu}={ }_{b-\mu} \nabla^{-1}\left(-\frac{\nabla(b-t) \frac{\mu+1}{}}{\mu+1}\right) \\
& =\sum_{s=t+1}^{b-\mu}(s-t-1)^{\underline{0}}\left(-\frac{\nabla(b-s) \frac{\mu+1}{\mu}}{\mu+1}\right) \\
& =\sum_{s=t+1}^{b-\mu}\left(\frac{(b-s+1)^{\frac{\mu+1}{}}}{\mu+1}-\frac{(b-s)^{\frac{\mu+1}{}}}{\mu+1}\right) \\
& =\frac{(b-t) \frac{\mu+1}{\mu}}{\mu+1}-\frac{\mu \frac{\mu+1}{\mu+1}}{\mu+1} \\
& =\mu^{-1}(b-t) \underline{\mu+1} \text {. }
\end{aligned}
$$

For $v \in(0,1) \cup(1,+\infty)$, define for $t \in_{b-\mu-\nu} \mathbb{N}$ the functions

$$
g_{1}(t)={ }_{b-\mu} \nabla^{-v}(b-t)^{\underline{\mu}}
$$

and

$$
g_{2}(t)=\mu^{\underline{-v}}(b-t) \underline{\mu+v} .
$$

We will show that both $g_{1}$ and $g_{2}$ solve the well-posed, first-order initial value problem

$$
\left\{\begin{array}{l}
(b-t-\mu-v+1) \nabla g(t)+(\mu+v) g(t)=0, \quad t \in{ }_{b-\mu-v} \mathbb{N} \\
g(b-\mu-v)=\Gamma(\mu+1)
\end{array}\right.
$$

Since

$$
\begin{aligned}
g_{1}(b-\mu-v) & =\left.\frac{1}{\Gamma(\nu)} \sum_{s=t+v}^{b-\mu}(s-t-1)^{\frac{\nu-1}{}}(b-s)^{\underline{\mu}}\right|_{t=b-\mu-\nu} \\
& =\frac{1}{\Gamma(\nu)} \sum_{s=b-\mu}^{b-\mu}(s-b+\mu+v-1)^{\frac{v-1}{}}(b-s)^{\underline{\mu}} \\
& =\frac{1}{\Gamma(v)}(v-1)^{\frac{v-1}{\mu}} \mu^{\underline{\mu}} \\
& =\Gamma(\mu+1)
\end{aligned}
$$

and

$$
g_{2}(b-\mu-v)=\mu \underline{-v}(\mu+v) \underline{\mu+v}=\Gamma(\mu+1),
$$

both $g_{1}$ and $g_{2}$ satisfy the initial condition in (2.8). 
An effort is required to show that $g_{1}$ satisfies the difference equation in (2.8). For $t \in$ $b-\mu-\nu, \mathbb{N}$,

$$
\begin{aligned}
\nabla g_{1}(t) & =\nabla\left(\frac{1}{\Gamma(\nu)} \sum_{s=t+\nu}^{b-\mu}(s-t-1)^{\underline{\nu-1}}(b-s)^{\underline{\mu}}\right) \\
& =\frac{1}{\Gamma(\nu)}\left(\sum_{s=t+\nu}^{b-\mu}(s-t-1)^{\frac{\nu-1}{}}(b-s)^{\underline{\mu}}-\sum_{s=t+\nu-1}^{b-\mu}(s-t)^{\underline{\nu-1}}(b-s)^{\underline{\mu}}\right) \\
& =\frac{-(\nu-1)}{\Gamma(v)} \sum_{s=t+\nu}^{b-\mu}(s-t-1)^{\underline{\nu-2}}(b-s)^{\underline{\mu}}-(b-t+1-v)^{\underline{\mu}} .
\end{aligned}
$$

Also, we may manipulate $g_{1}$ directly to obtain

$$
\begin{aligned}
& g_{1}(t)=\frac{1}{\Gamma(\nu)} \sum_{s=t+\nu}^{b-\mu}(s-t-1)^{\underline{\nu-1}}(b-s)^{\underline{\mu}} \\
& =\frac{1}{\Gamma(v)} \sum_{s=t+v}^{b-\mu}(s-t-1-(v-2))(s-t-1)^{\frac{\nu-2}{}(b-s)^{\underline{\mu}}} \\
& =\frac{1}{\Gamma(v)} \sum_{s=t+v}^{b-\mu}[(b-t-(\mu+v)+1)-(b-s-\mu)](s-t-1)^{\frac{\nu-2}{}(b-s)^{\mu}} \\
& =\frac{b-t-\mu-v+1}{\Gamma(\nu)} \sum_{s=t+\nu}^{b-\mu}(s-t-1)^{\underline{\nu-2}}(b-s)^{\underline{\mu}} \\
& -\frac{1}{\Gamma(\nu)} \sum_{s=t+v}^{b-\mu}(b-s-\mu)(s-t-1)^{\frac{\nu-2}{}}(b-s)^{\mu} \\
& =\frac{b-t-\mu-v+1}{\Gamma(v)} \sum_{s=t+v}^{b-\mu}(s-t-1)^{\frac{\nu-2}{2}}(b-s)^{\underline{\mu}} \\
& -\frac{1}{\Gamma(v)} \sum_{s=t+v}^{b-\mu}(s-t-1)^{\frac{v-2}{(b}}(b-s) \underline{\mu+1} \\
& =h(t)-k(t),
\end{aligned}
$$

where

$$
\left\{\begin{array}{l}
h(t):=\frac{b-t-\mu-v+1}{\Gamma(v)} \sum_{s=t+v}^{b-\mu}(s-t-1)^{\underline{\nu-2}}(b-s)^{\underline{\mu}}, \\
k(t):=\frac{1}{\Gamma(v)} \sum_{s=t+v}^{b-\mu}(s-t-1)^{\underline{\nu}-2}(b-s)^{\underline{\mu+1}} .
\end{array}\right.
$$

Summing $k(t)$ by parts, we obtain

$$
\begin{aligned}
k(t) & =\frac{1}{\Gamma(v)} \sum_{s=t+v}^{b-\mu}(s-t-1) \frac{\nu-2}{(b-s) \underline{\mu+1}} \\
& =\frac{1}{\Gamma(\nu)} \sum_{s=t+v}^{b-\mu}(b-s) \frac{\mu+1}{\underline{ }} \triangle_{s}\left(\frac{(s-t-1)^{\frac{\nu-1}{}}}{v-1}\right)
\end{aligned}
$$




$$
\begin{aligned}
& =\left.\frac{1}{\Gamma(\nu)}(b-s) \frac{\mu+1}{\frac{(s-t)^{\nu-1}}{\nu-1}}\right|_{s=t+\nu} ^{b-\mu+1}+\frac{1}{\Gamma(\nu)} \sum_{s=t+\nu}^{b-\mu} \frac{(s-t)^{\frac{\nu-1}{}}}{v-1}(\mu+1)(b-s-1)^{\mu} \\
& =\frac{1}{v-1}\left[\frac{\mu+1}{\Gamma(v)} \sum_{s=t+v}^{b-\mu}(s-t)^{\underline{\nu-1}}(b-s-1)^{\underline{\mu}}-(b-t-v)^{\underline{\mu+1}}\right] \\
& =\frac{1}{v-1}\left[\frac{\mu+1}{\Gamma(v)} \sum_{u=t+v+1}^{b-\mu+1}(u-t-1)^{\frac{v-1}{(}}(b-u)^{\underline{\mu}}-(b-t-v)^{\underline{\mu+1}}\right] \\
& =\frac{1}{v-1}\left[\frac{\mu+1}{\Gamma(v)} \sum_{s=t+v}^{b-\mu}(s-t-1)^{\frac{v-1}{}(b-s)}\right)^{\mu} \\
& \left.-\frac{\mu+1}{\Gamma(v)}(v-1)^{\frac{v-1}{}}(b-t-v)^{\mu}-(b-t-v) \underline{\mu+1}\right] \\
& =\frac{1}{v-1}\left[\frac{\mu+1}{\Gamma(v)} \sum_{s=t+v}^{b-\mu}(s-t-1)^{\left.\frac{v-1}{(}(b-s)^{\underline{\mu}}-(b-t-v+1) \frac{\mu+1}{}\right] .}\right.
\end{aligned}
$$

It follows from the above work that

$$
\begin{aligned}
& -(b-t-\mu-v+1) \nabla g_{1}(t)=(v-1) h(t)+(b-t-v+1) \stackrel{\mu+1}{,} \\
& (\mu+1) g_{1}(t)-(v-1) k(t)=(b-t-v+1) \stackrel{\mu+1}{ } .
\end{aligned}
$$

Hence,

$$
(b-t-\mu-v+1) \nabla g_{1}(t)+(\mu+v) g_{1}(t)=0 \text {. }
$$

Finally, $g_{2}$ also satisfies the difference equation (2.8):

$$
\begin{aligned}
& (b-t-\mu-v+1) \nabla g_{2}(t)=(b-t-\mu-v+1) \mu \frac{-v}{-}\left[(b-t) \underline{\mu+v}-(b-t+1) \frac{\mu+v}{v}\right] \\
& =-(b-t-\mu-v+1) \mu^{\underline{-v}}(\mu+v)(b-t) \underline{\mu+v-1} \\
& =-(\mu+v) g_{2}(t) \text {. }
\end{aligned}
$$

By the uniqueness of the solutions to the well-posed initial value problem (2.8), we conclude that $g_{1} \equiv g_{2}$ on ${ }_{b-\mu-\nu} \mathbb{N}$.

We next employ (2.5) and (2.6) to show (2.7) follows. For $t \in{ }_{b-\mu-N+v} \mathbb{N}$,

$$
\begin{aligned}
& { }_{b-\mu} \nabla^{\nu}(b-t)^{\underline{\mu}}=(-1)^{N} \nabla^{N}{ }_{b-\mu} \nabla^{-(N-v)}(b-t)^{\underline{\mu}} \\
& =(-1)^{N} \nabla^{N}(\mu \underline{-(N-v)}(b-t) \underline{\mu+N-v}) \\
& =(-1)^{N} \nabla^{N}\left(\frac{\Gamma(\mu+1)}{\Gamma(\mu+1+N-v)}(b-t) \frac{\mu+N-v}{}\right) \\
& =(-1)^{N} \nabla^{N-1}\left(\frac{\Gamma(\mu+1)}{\Gamma(\mu+1+N-v)}\left((b-t) \frac{\mu+N-v}{\left.\left.\frac{1+N}{2}-t+1\right) \frac{\mu+N-v}{}\right)}\right)\right. \\
& =(-1)^{N-1} \nabla^{N-1}\left(\frac{\Gamma(\mu+1)}{\Gamma(\mu+N-v)}(b-t) \frac{\mu+N-v-1}{}\right)
\end{aligned}
$$




$$
\begin{aligned}
& =\frac{\Gamma(\mu+1)}{\Gamma(\mu+1-v)}(b-t) \frac{\mu-v}{\underline{\nu}} \\
& =\mu^{\underline{v}}(b-t) \underline{\mu-v} .
\end{aligned}
$$

Theorem 2 Let $f:{ }_{b} \mathbb{N} \rightarrow \mathbb{R}$ be given and suppose $\mu, v>0$. Then

$$
{ }_{b-\mu} \nabla^{-\nu}{ }_{b} \nabla^{-\mu} f(t)={ }_{b} \nabla^{-\mu-\nu} f(t)={ }_{b-\nu} \nabla^{-\mu}{ }_{b} \nabla^{-v} f(t), \quad t \in{ }_{b-\mu-\nu} \mathbb{N} .
$$

Proof Suppose $f:{ }_{b} \mathbb{N} \rightarrow \mathbb{R}$ and $\mu, v>0$. Then for $t \in_{b-\mu-v} \mathbb{N}$,

$$
\begin{aligned}
& { }_{b-\mu} \nabla^{-v}{ }_{b} \nabla^{-\mu} f(t)=\frac{1}{\Gamma(\nu)} \sum_{s=t+\nu}^{b-\mu}(s-t-1)^{\frac{v-1}{2}} \frac{1}{\Gamma(\mu)} \sum_{u=s+\mu}^{b}(u-s-1)^{\mu-1} f(u) \\
& =\frac{1}{\Gamma(\nu) \Gamma(\mu)} \sum_{s=t}^{b-(\mu+\nu)} \sum_{u=s+\mu+v}^{b}(s+v-t-1)^{\frac{\nu-1}{2}}(u-s-v-1)^{\mu-1} f(u) \\
& =\frac{1}{\Gamma(v) \Gamma(\mu)} \sum_{s=t}^{b-\mu-v} \sum_{r=s}^{b-\mu-v}(s+v-t-1)^{\frac{\nu-1}{}(r+\mu-s-1)^{\mu-1}} f(r+\mu+v) \\
& =\frac{1}{\Gamma(v) \Gamma(\mu)} \sum_{r=t}^{b-\mu-v} \sum_{s=t}^{r}(s+v-t-1)^{\frac{\nu-1}{}}(r+\mu-s-1) \frac{\mu-1}{f}(r+\mu+v) \\
& =\frac{1}{\Gamma(v) \Gamma(\mu)} \sum_{r=t}^{b-\mu-v} f(r+\mu+v) \\
& \times \sum_{s=t-v+\nu}^{r}(s-(t-v)-1)^{\frac{\nu-1}{(}(r+\mu-s-1)^{\mu-1}} \\
& =\left.\frac{1}{\Gamma(\mu)} \sum_{r=t}^{b-\mu-v} f(r+\mu+v)_{(r+\mu-1)-(\mu-1)} \nabla^{-v}(r+\mu-1-t) \frac{\mu-1}{}\right|_{t-v} \\
& =\frac{1}{\Gamma(\mu)} \sum_{r=t}^{b-\mu-v} f(r+\mu+v)(\mu-1)^{-\underline{v}}(r+\mu-1-(t-v))^{\mu+\nu-1}
\end{aligned}
$$

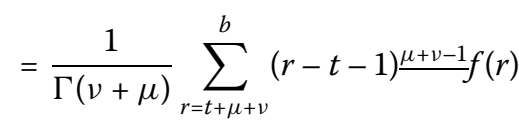

$$
\begin{aligned}
& ={ }_{b} \nabla^{-\mu-v} f(t) \text {. }
\end{aligned}
$$

Since $v$ and $\mu$ are arbitrary, we conclude more generally that

$$
{ }_{b-\mu} \nabla_{b}^{-v} \nabla^{-\mu} f(t)={ }_{b} \nabla^{-\mu-v} f(t)={ }_{b-\nu} \nabla_{b}^{-\mu} \nabla^{-v} f(t), \quad t \in{ }_{b-\mu-\nu} \mathbb{N} .
$$

Lemma 2 Let $f:{ }_{b} \mathbb{N} \rightarrow \mathbb{R}$ be given. For any $k \in \mathbb{N}_{0}$ and $\mu>0$ with $M-1<\mu \leq M$, we have

$$
\begin{aligned}
& { }_{b-\mu} \nabla^{k}\left({ }_{b} \nabla^{-\mu} f(t)\right)={ }_{b} \nabla^{k-\mu} f(t), \quad t \in{ }_{b-\mu} \mathbb{N}, \\
& { }_{b-M+\mu} \nabla^{k}\left({ }_{b} \nabla^{\mu} f(t)\right)={ }_{b} \nabla^{k+\mu} f(t), \quad t \in \in_{b-M+\mu} \mathbb{N} .
\end{aligned}
$$

Proof Let $f, \mu, M$, and $k$ be as given in the statement of the lemma. We first prove (2.9). We consider two cases. 
Case 1. $\mu=M$.

Observe that for $t \in{ }_{b-1} \mathbb{N}$,

$$
\nabla_{b} \nabla^{-1} f(t)=\nabla\left(\sum_{s=t+1}^{b} f(s)\right)=-f(t) .
$$

Furthermore, for any $k \in \mathbb{N}^{+}$and $t \in_{b-k} \mathbb{N}$, by Theorem 2 , we have

$$
\begin{aligned}
\nabla_{b}^{k} \nabla^{-k} f(t)= & \nabla^{k-1}\left(\nabla_{b-k+1} \nabla^{-1}\left({ }_{b} \nabla^{-(k-1)} f(t)\right)\right) \\
= & -\nabla^{k-1}\left({ }_{b} \nabla^{-(k-1)} f(t)\right) \\
& \vdots \\
= & (-1)^{k} f(t) .
\end{aligned}
$$

Therefore, for any $t \in \in_{b-M} \mathbb{N}$, we obtain

$$
\begin{aligned}
{ }_{b-M} \nabla_{b}^{k} \nabla^{-M} f(t) & =(-1)^{k} \nabla_{b}^{k} \nabla^{-M} f(t) \\
& =(-1)^{k} \nabla^{k-M} \nabla^{M}{ }_{b} \nabla^{-M} f(t) \\
& =(-1)^{k} \nabla^{k-M} f(t) \\
& ={ }_{b} \nabla^{k-M} f(t), \quad \text { if } k \geq M ; \\
{ }_{b-M} \nabla^{k}{ }_{b} \nabla^{-M} f(t) & =(-1)^{k} \nabla^{k}{ }_{b} \nabla^{-M} f(t) \\
& =(-1)^{k} \nabla_{b-M+k}^{k} \nabla^{-k}{ }_{b} \nabla^{-(M-k)} f(t) \\
& ={ }_{b} \nabla^{k-M} f(t), \quad \text { if } k<M .
\end{aligned}
$$

Case 2. $M-1<\mu<M$.

$$
\begin{aligned}
\nabla_{b} \nabla^{-\mu} f(t) & =\nabla \frac{1}{\Gamma(\mu)} \sum_{s=t+\mu}^{b}(s-t-1) \frac{\mu-1}{f} f(s) \\
& =\frac{1}{\Gamma(\mu)}\left[\sum_{s=t+\mu}^{b}(s-t-1) \frac{\mu-1}{f} f(s)-\sum_{s=t+\mu-1}^{b}(s-t) \frac{\mu-1}{f} f(s)\right] \\
& =-\frac{1}{\Gamma(\mu-1)} \sum_{s=t+\mu-1}^{b}(s-t-1) \frac{\mu-2}{f} f(s) \\
& =-{ }_{b} \nabla^{1-\mu} f(t) .
\end{aligned}
$$

Repeating the above process, we may see that (2.9) holds.

Next, by (2.9), we get

$$
\begin{aligned}
{ }_{b-M+\mu} \nabla^{k}\left({ }_{b} \nabla^{\mu} f(t)\right) & =(-1)^{k} \nabla^{k}(-1)^{M} \nabla^{M}\left({ }_{b} \nabla^{-(M-\mu)} f(t)\right) \\
& =(-1)^{k+M} \nabla^{k+M}\left({ }_{b} \nabla^{-(M-\mu)} f(t)\right) \\
& ={ }_{b-M+\mu} \nabla^{k+M}\left({ }_{b} \nabla^{-(M-\mu)} f(t)\right)={ }_{b} \nabla^{k+\mu} f(t),
\end{aligned}
$$

and hence (2.10) holds. 
Theorem 3 Let $f:{ }_{b} \mathbb{N} \rightarrow \mathbb{R}$ be given and suppose $\mu, v>0$ with $N-1<v<N$. Then

$$
{ }_{b-\mu} \nabla_{b}^{v} \nabla^{-\mu} f(t)={ }_{b} \nabla^{\nu-\mu} f(t), \quad t \in{ }_{b-\mu-N+v} \mathbb{N}
$$

Proof Let $f, v, N$, and $\mu$ be given as in the statement of the theorem and let $t \in{ }_{b-\mu-N+v} \mathbb{N}$. Then by Theorem 2 and Lemma 2, we have

$$
\begin{aligned}
{ }_{b-\mu} \nabla_{b}{ }_{b} \nabla^{-\mu} f(t) & =(-1)^{N} \nabla^{N}{ }_{b-\mu} \nabla^{-(N-v)}{ }_{b} \nabla^{-\mu} f(t) \\
& =(-1)^{N} \nabla^{N}{ }_{b} \nabla^{-(N-v)-\mu} f(t) \\
& ={ }_{b} \nabla^{v-\mu} f(t) .
\end{aligned}
$$

Theorem 4 Let $f:{ }_{b} \mathbb{N} \rightarrow \mathbb{R}$ be given and suppose $k \in \mathbb{N}_{0}$ be given. Then for $t \in{ }_{b-v} \mathbb{N}$,

$$
{ }_{b} \nabla^{-v}{ }_{b} \nabla^{k} f(t)={ }_{b} \nabla^{k-v} f(t)-\sum_{j=0}^{k-1} \frac{{ }_{b} \nabla^{j} f(b)}{\Gamma(v-k+j+1)}(b-t) \frac{v-k+j}{} .
$$

Moreover, if $\mu>0$ with $M-1<\mu<M$, then for $t \in_{b-M+\mu-\nu} \mathbb{N}$,

$$
{ }_{b-M+\mu} \nabla^{-v}{ }_{b} \nabla^{\mu} f(t)={ }_{b} \nabla^{\mu-v} f(t)-\sum_{j=0}^{M-1} \frac{{ }_{b} \nabla^{j-M+\mu} f(b-M+\mu)}{\Gamma(v-M+j+1)}(b-M+\mu-t) \frac{\nu-M+j}{} .
$$

Proof We first consider (2.12). Let $k \in \mathbb{N}_{0}$ be given. Then

$$
\begin{aligned}
& { }_{b} \nabla^{-v}{ }_{b} \nabla^{k} f(t)=(-1)^{k}{ }_{b} \nabla^{-v} \nabla^{k} f(t) \\
& =(-1)^{k} \frac{1}{\Gamma(\nu)} \sum_{s=t+v}^{b}(s-t-1)^{\frac{\nu-1}{}} \nabla^{k} f(s) \\
& =(-1)^{k} \frac{1}{\Gamma(\nu)}\left(\sum_{s=t+v}^{b}(s-t-1)^{\frac{\nu-1}{2}} \triangle\left(\nabla^{k-1} f(s-1)\right)\right) \\
& =(-1)^{k} \frac{1}{\Gamma(v)}\left(\left.(s-t-1) \frac{v-1}{} \nabla^{k-1} f(s-1)\right|_{s=t+v} ^{b+1}\right. \\
& \left.-\sum_{s=t+v}^{b}(v-1)(s-t-1)^{\frac{v-2}{2}} \nabla^{k-1} f(s)\right) \\
& =(-1)^{k}\left(-\frac{1}{\Gamma(v-1)} \sum_{s=t+v-1}^{b}(s-t-1) \frac{v-2}{2} \nabla^{k-1} f(s)+\frac{\nabla^{k-1} f(b)}{\Gamma(v)}(b-t) \frac{v-1}{}\right) \\
& =(-1)^{k-1} \frac{1}{\Gamma(v-1)} \sum_{s=t+v-1}^{b}(s-t-1)^{\frac{\nu-2}{2}} \nabla^{k-1} f(s)-\frac{{ }_{b} \nabla^{k-1} f(b)}{\Gamma(v)}(b-t)^{\frac{\nu-1}{}} \\
& =(-1)^{k-2} \frac{1}{\Gamma(v-2)} \sum_{s=t+v-2}^{b}(s-t-1)^{\frac{v-3}{2}} \nabla^{k-2} f(s) \\
& -\frac{{ }_{b} \nabla^{k-2} f(b)}{\Gamma(\nu-1)}(b-t)^{\frac{\nu-2}{2}}-\frac{{ }_{b} \nabla^{k-1} f(b)}{\Gamma(\nu)}(b-t)^{\frac{\nu-1}{}}
\end{aligned}
$$




$$
\begin{aligned}
= & \frac{1}{\Gamma(v-k)} \sum_{s=t+v-k}^{b}(s-t-1)^{\nu-k-1} f(s) \\
& -\sum_{j=0}^{k-1} \frac{{ }_{b} \nabla^{j} f(b)}{\Gamma(v-k+j+1)}(b-t) \stackrel{v-k+j}{.}
\end{aligned}
$$

We next consider (2.13). Suppose that $\nu, \mu>0$ with $M-1<\mu \leq M$. Defining

$$
g(t):={ }_{b} \nabla^{-(M-\mu)} f(t) \quad \text { and } \quad a:=b-M+\mu,
$$

where $a$ is the domain of the first point $g$, we have, for $t \in{ }_{b-M+\mu-\nu} \mathbb{N}$,

$$
\begin{aligned}
{ }_{b-M+\mu} \nabla^{-v}{ }_{b} \nabla^{\mu} f(t)= & { }_{b-M+\mu} \nabla^{-\nu}(-1)^{M} \nabla^{M}{ }_{b} \nabla^{-(M-\mu)} f(t) \\
= & (-1)_{b-M+\mu}^{M} \nabla^{-\nu} \nabla^{M} g(t) \\
= & { }_{b-M+\mu} \nabla^{-\nu}{ }_{b-M+\mu} \nabla^{M} g(t) \\
= & { }_{b-M+\mu} \nabla^{M-v} g(t)-\sum_{j=0}^{M-1} \frac{b-M+\mu}{\Gamma(v-M+j+1)}(a-t)^{\nu-M+j} \\
= & { }_{b-M+\mu} \nabla^{M-v}{ }_{b} \nabla^{-(M-\mu)} f(t) \\
& -\sum_{j=0}^{M-1} \frac{b-M+\mu}{\Gamma(v-M+j+1)}(a-t)_{b} \nabla^{-(M-\mu)} f(a) \\
= & { }_{b} \nabla^{\mu-v} f(t)-\sum_{j=0}^{M-1} \frac{{ }_{b} \nabla^{-(M-\mu)+j} f(b-M+\mu)}{\Gamma(v-M+j+1)}(b-M+\mu-t) \frac{v-M+j}{} .
\end{aligned}
$$

Theorem 5 Letf $:{ }_{b} \mathbb{N} \rightarrow \mathbb{R}$ be given and suppose $\mu, v>0$ with $N-1<v \leq N$ and $M-1<$ $\mu \leq M$. Then for $t \in{ }_{b-M+\mu-N+\nu} \mathbb{N}$,

$$
{ }_{b-M+\mu} \nabla^{v}{ }_{b} \nabla^{\mu} f(t)={ }_{b} \nabla^{\mu+v} f(t)-\sum_{j=0}^{M-1} \frac{{ }_{b} \nabla^{j-M+\mu} f(b-M+\mu)}{\Gamma(-v-M+j+1)}(b-M+\mu-t) \stackrel{-v-M+j}{ },
$$

where in agreement with both rule (2.14) and the standard convention on $t$, the terms in the summation vanish in the case $v \in \mathbb{N}_{0}$.

Proof Let $f, v$, and $\mu$ be given as in the statement of the theorem. Recall that Lemma 2 proves (2.14) in the case when $v=N$. On the other hand, if $N-1<v<N$, then by Theorem 4 , we have for $t \in{ }_{b-M+\mu-N+\nu} \mathbb{N}$,

$$
\begin{aligned}
{ }_{b-M+\mu} \nabla_{b}{ }_{b} \nabla^{\mu} f(t)= & (-1)^{N} \nabla_{b-M+\mu}^{N} \nabla^{-(N-v)}{ }_{b} \nabla^{\mu} f(t) \\
= & (-1)^{N} \nabla^{N}\left[{ }_{b} \nabla^{-N+v+\mu} f(t)-\sum_{j=0}^{M-1} \frac{{ }_{b} \nabla^{j-M+\mu} f(b-M+\mu)}{\Gamma(N-v-M+j+1)}\right. \\
& \left.\times(b-M+\mu-t) \frac{N-\nu-M+j}{}\right]
\end{aligned}
$$




$$
\begin{aligned}
= & { }_{b} \nabla^{\mu+v} f(t)-\sum_{j=0}^{M-1} \frac{{ }_{b} \nabla^{j-M+\mu} f(b-M+\mu)}{\Gamma(N-v-M+j+1)} \\
& \times(-1)^{N} \nabla^{N}(b-M+\mu-t) \underline{N-v-M+j} \\
= & { }_{b} \nabla^{\mu+v} f(t)-\sum_{j=0}^{M-1} \frac{{ }_{b} \nabla^{j-M+\mu} f(b-M+\mu)}{\Gamma(-v-M+j+1)}(b-M+\mu-t)^{-v-M+j} .
\end{aligned}
$$

\section{Application}

Theorem 6 Let $f:{ }_{b} \mathbb{N} \rightarrow \mathbb{R}$ and $v>0$ be given with $N-1<v<N$, consider the initial value problem of the $v$ th right fractional difference equation

$$
\left\{\begin{array}{l}
{ }_{b} \nabla^{v} y(t)=f(t), \quad t \in{ }_{b} \mathbb{N} \\
\nabla^{i} y(b)=A_{i}, \quad i=0,1, \ldots, N-1, A_{i} \in \mathbb{R} .
\end{array}\right.
$$

The general solution to (3.1) is

$$
y(t)={ }_{b-N+\nu} \nabla^{-v} f(t)+\sum_{i=0}^{N-1} \alpha_{i}(b-N+v-t)^{i+\nu-N},
$$

where $\left\{\alpha_{j}\right\}_{j=0}^{N-1}$ are $N$ real constants. Moreover, the unique solution to (3.1) is (3.2) with particular constants,

$$
\begin{aligned}
& \alpha_{j}=\frac{1}{\Gamma(v-N+j+1)} \sum_{k=0}^{j} \sum_{i=0}^{j-k}(-1)^{k+i}\left(\begin{array}{c}
j-N+v \\
k
\end{array}\right)\left(\begin{array}{c}
j-k \\
i
\end{array}\right) A_{i}, \\
& \left(\begin{array}{l}
\alpha \\
\beta
\end{array}\right)=\frac{\Gamma(\alpha+1)}{\Gamma(\beta+1) \Gamma(\alpha-\beta+1)} .
\end{aligned}
$$

Proof By Theorem 4, we have

$$
y(t)={ }_{b-N+v} \nabla^{-v} f(t)+\sum_{j=0}^{N-1} \frac{{ }_{b} \nabla^{j-N+v} y(b-N+v)}{\Gamma(v-N+j+1)}(b-N+v-t) \frac{v-N+j}{} .
$$

We have

$$
\begin{aligned}
{ }_{b} \nabla^{j-N+v} y(b-N+v)= & \frac{1}{\Gamma(N-v-j)} \sum_{s=b-j}^{b}(s-b+N-v-1) \frac{-j+N-v-1}{d} y(s) \\
= & \frac{1}{\Gamma(N-v-j)} \sum_{k=0}^{j}(k-j+N-v-1) \frac{-j+N-v-1}{d} y(b-j+k) \\
= & \frac{1}{\Gamma(N-v-j)} \sum_{k=0}^{j} \frac{\Gamma(k-j+N-v)}{\Gamma(k+1)} y(b-j+k) \\
= & \sum_{k=0}^{j} \frac{(k-j+N-v-1)(k-j+N-v-2) \cdots(N-v-j)}{\Gamma(k+1)} \\
& \times y(b-j+k) \\
= & \sum_{k=0}^{j}(-1)^{k}\left(\begin{array}{c}
v+j-N \\
k
\end{array}\right) y(b-j+k)
\end{aligned}
$$




$$
\begin{aligned}
& =\sum_{k=0}^{j}(-1)^{k}\left(\begin{array}{c}
v+j-N \\
k
\end{array}\right) \sum_{i=0}^{j-k}(-1)^{i}\left(\begin{array}{c}
j-k \\
i
\end{array}\right) \nabla^{i} y(b) \\
& =\sum_{k=0}^{j} \sum_{i=0}^{j-k}(-1)^{i+k}\left(\begin{array}{c}
v+j-N \\
k
\end{array}\right)\left(\begin{array}{c}
j-k \\
i
\end{array}\right) A_{i} .
\end{aligned}
$$

Therefore, $\alpha_{j}=\frac{1}{\Gamma(v-N+j+1)} \sum_{k=0}^{j} \sum_{i=0}^{j-k}(-1)^{i+k}\left(\begin{array}{c}v+j-N \\ k\end{array}\right)\left(\begin{array}{c}j-k \\ i\end{array}\right) A_{i}$.

We consider two-point boundary value problem

$$
\left\{\begin{array}{l}
{ }_{b} \nabla^{v} y(t)+f(t-v+1, y(t-v+1))=0, \quad t \in[v-1, b+v-1]_{\mathbb{N}_{v-1}}, \\
y(-1)=y(b)=0
\end{array}\right.
$$

where $f:[0, b]_{\mathbb{N}_{0}} \times \mathbb{R} \rightarrow \mathbb{R}$ is a continuous function and $v \in(1,2], b \in \mathbb{N}, b>2$.

By Theorem 4, we have

$$
\begin{aligned}
& y(t)={ }_{b-2-v} \nabla^{-v} f(t-v+1, y(t-v+1)) \\
& +C_{1}(b-2+v-t)^{\frac{v-1}{}}+C_{2}(b-2+v-t)^{\frac{v-2}{}}, \\
& =-\frac{1}{\Gamma(v)} \sum_{s=t+v}^{b-2+v}(s-t-1) \frac{v-1}{f} f(s-v+1, y(s-v+1)) \\
& +C_{1}(b-2+v-t)^{\frac{v-1}{}}+C_{2}(b-2+v-t)^{\frac{v-2}{2}}, \\
& y(b)=C_{2} \Gamma(v-1)=0, \quad C_{2}=0, \\
& y(-1)=-\frac{1}{\Gamma(\nu)} \sum_{s=v-1}^{b+v-2} s \frac{v-1}{f} f(s-v+1, y(s-v+1))+C_{1}(b-1+v)^{\frac{\nu-1}{}}=0, \\
& C_{1}=\frac{1}{(b+v-1) \frac{v-1}{\Gamma} \Gamma(v)} \sum_{s=v-1}^{b+v-2} s^{\frac{v-1}{}} f(s-v+1, y(s-v+1)) \\
& y(t)=\sum_{s=v-1}^{b+v-2} G(t, s) f(s-v+1, y(s-v+1)),
\end{aligned}
$$

where

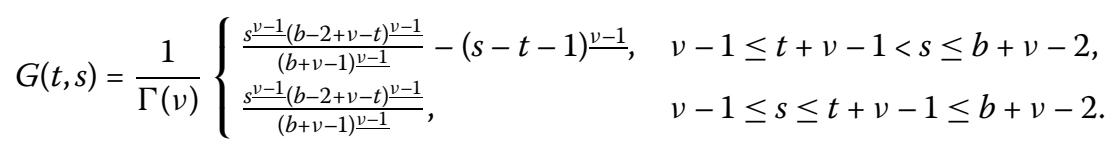

Theorem 7 The Green's function $G(t, s)$ satisfies the following conditions:

(i) $G(t, s)>0$ for $t \in[0, b-1]_{\mathbb{N}_{0}}$ and $s \in[v, b+v-2]_{\mathbb{N}_{\nu}}$;

(ii) $\max _{t \in[0, b]_{\mathbb{N}_{0}}} G(t, s)=G(s-v+1, s)$, for $s \in[v, b+v-2]_{\mathbb{N}_{v}}$;

(iii) there exists a positive number $\gamma \in(0,1)$ such that

$$
\begin{aligned}
& \min _{t \in\left[\frac{b}{4}, \frac{3 b}{4}\right]} G(t, s) \geq \gamma \max _{t \in[-1, b]_{\mathbb{N}_{-1}}} G(t, s)=\gamma G(s-v+1, s), \text { for } \\
& s \in[v-1, b+v-2]_{\mathbb{N}_{v-1}} .
\end{aligned}
$$

Proof (i) One can see that $\triangle_{t} G(t, s)<0$ for $v-1 \leq s \leq t+v-1$, and $\triangle_{t} G(t, s)>0$ for $v-1 \leq t+v-1<s$. Indeed, for $v-1 \leq t+v-1<s$, we have

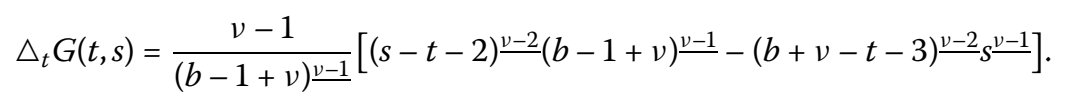


Thus, $\triangle_{t} G(t, s)>0$ if and only if

$$
\frac{(s-t-2)^{\frac{\nu-2}{}}(b-1+v)^{\frac{\nu-1}{}}}{(b+v-t-3)^{\frac{\nu-2}{2}} s^{\underline{\nu}-1}}>1 .
$$

The inequality follows from the fact that $t^{\underline{\alpha}}$ is increasing and $t^{-\alpha}$ is decreasing if $0<\alpha \leq 1$. Since

$$
G(-1, s)=G(b, s)=0
$$

and

$$
\begin{aligned}
& G(s-v+1, s)=\frac{s^{\frac{\nu-1}{}}(b+v-2-s+v-1)^{\underline{v-1}}}{(b+v-1)^{\underline{v-1}}} \\
& =\frac{s^{\underline{\nu-1}}(b+2 v-s-3)^{\underline{\nu-1}}}{(b+v-1) \underline{\underline{v-1}}}>0, \quad s \in[v-1, b+v-2]_{\mathbb{N}_{v-1}},
\end{aligned}
$$

(i), (ii) are proved.

Next, we prove the (iii). Clearly,

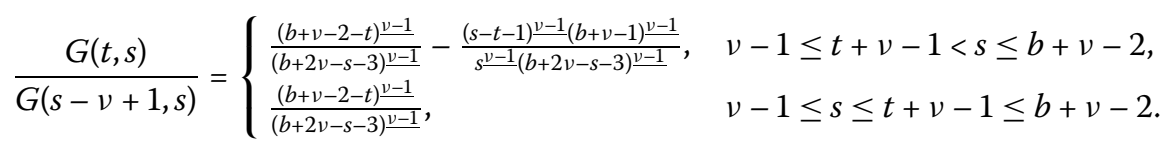

For $v-1 \leq s \leq t+v-1 \leq b+v-2$ and $\frac{b}{4} \leq t \leq \frac{3 b}{4}$,

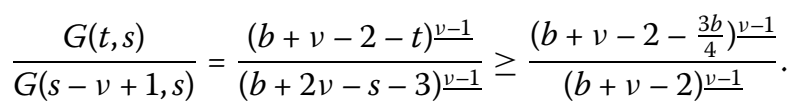

For $v-1 \leq t+v-1<s \leq b+v-2$ and $\frac{b}{4} \leq t \leq \frac{3 b}{4}$, we know that $G(t, s)$ is increasing with respect to $t$, hence we have

$$
\begin{aligned}
& \frac{G(t, s)}{G(s-v+1, s)} \geq \frac{\left(b+v-2-\frac{b}{4}\right)^{\frac{v-1}{w}}}{(b+2 v-s-3) \underline{\underline{v-1}}}-\frac{\left(s-\frac{b}{4}-1\right)^{v-1}(b+v-1)^{\underline{v-1}}}{s^{\underline{v-1}}(b+2 v-s-3) \underline{\underline{v-1}}} \\
& =\frac{1}{(b+2 v-s-3)^{\underline{v-1}}} \\
& \times\left[\left(\frac{3 b}{4}+v-2\right)^{\frac{v-1}{2}}-\frac{1}{s \underline{v-1}}\left(s-\frac{b}{4}-1\right)^{\frac{v-1}{(}}(b+v-1)^{\frac{v-1}{}}\right] \\
& \geq \frac{1}{(b+v-2)^{\underline{v-1}}}\left[\left(\frac{3 b}{4}+v-2\right)^{\underline{v-1}}-\frac{\left(\frac{3 b}{4}+v-3\right)^{\frac{v-1}{v-1}}}{(b+v-2)^{\underline{v-1}}}(b+v-1)^{\frac{v-1}{2}}\right] \\
& >\frac{1}{(b+v-2)^{\underline{v-1}}}\left[\left(\frac{3 b}{4}+v-2\right)^{\underline{v-1}}-\frac{\left(\frac{3 b}{4}+v-2\right)^{\underline{v-1}}}{(b+v-1)^{\frac{v-1}{u}}}(b+v-1)^{\frac{v-1}{}}\right]=0 \text {, }
\end{aligned}
$$

since $\frac{\left(s-\frac{b}{4}-1\right) \underline{v-1}}{s^{\underline{\nu}-1}}$ is increasing for $s$.

Thus

$$
\min _{t \in\left[\frac{b}{4}, \frac{3 b}{4}\right]} G(t, s) \geq \gamma \max _{t \in[-1, b]_{\mathbb{N}_{-1}}} G(t, s)=\gamma G(s-v+1, s),
$$


where

$$
\begin{aligned}
& \gamma=\min \left\{\frac{\left(\frac{b}{4}+v-2\right)^{\underline{v-1}}}{(b+v-2) \underline{v-1}},\right. \\
& \left.\frac{1}{(b+v-2) \underline{v-1}}\left[\left(\frac{3 b}{4}+v-2\right)^{\frac{v-1}{2}}-\frac{\left(\frac{3 b}{4}+v-3\right)^{\frac{v-1}{}}}{(b+v-2)^{\underline{v-1}}}(b+v-1)^{\frac{v-1}{1}}\right]\right\} \text {. }
\end{aligned}
$$

Below we shall employ the following fixed point result.

Lemma 3 (see [2]) Let $\mathbb{B}$ be a Banach space, and let $\mathcal{P} \subset \mathbb{B}$ be a cone. Assume $\Omega_{1}$ and $\Omega_{2}$ are open discs contained in $\mathbb{B}$ with $0 \in \Omega_{1}, \bar{\Omega}_{1} \subset \Omega_{2}$ and let $A: \mathcal{P} \cap\left(\bar{\Omega}_{2} \backslash \Omega_{1}\right) \rightarrow \mathcal{P}$ be a completely continuous operator such that, either

(i) $\|A y\| \leq\|y\|, y \in \mathcal{P} \cap \partial \Omega_{1}$ and $\|A y\| \geq\|y\|, y \in \mathcal{P} \cap \partial \Omega_{2}$ or

(ii) $\|A y\| \geq\|y\|, y \in \mathcal{P} \cap \partial \Omega_{1}$ and $\|A y\| \leq\|y\|, y \in \mathcal{P} \cap \partial \Omega_{2}$.

Then $A$ has least one fixed point in $\mathcal{P} \cap\left(\bar{\Omega}_{2} \backslash \Omega_{1}\right)$.

Clearly, finding a solution $y(t)$ of the FBVP (3.3) is equivalent to finding a solution of the summation equation (3.4).

For our purpose, define the Banach space $\mathcal{B}$ by

$$
\mathcal{B}=\left\{y:[0, b]_{\mathbb{N}_{0}} \rightarrow \mathbb{R}: y(-1)=y(b)=0\right\},
$$

with norm $\|y\|=\max _{t \in[-1, b]]_{\mathbb{N}_{-}}}|y(t)|$.

Let $\gamma$ be defined by (3.5) and define cones $\mathcal{P}$ and $\mathcal{P}_{0}$ in $\mathbb{B}$ by

$$
\begin{aligned}
& \mathcal{P}=\left\{y \in \mathbb{B}: y(t) \geq 0 \text { for } t \in[-1, b]_{\mathbb{N}_{-1}}\right\}, \\
& \mathcal{P}_{0}=\left\{y \in \mathcal{P}: \min _{t \in\left[\frac{b}{4}, \frac{3 b}{4}\right]} y(t) \geq \gamma\|y\|\right\} .
\end{aligned}
$$

Thus, $y$ is a solution of the boundary value problem (3.3) if and only if $y$ is a fixed point of the operator $T: \mathbb{B} \rightarrow \mathbb{B}$ defined by

$$
T y(t)=\sum_{s=v-1}^{b+v-2} G(t, s) f(s-v+1, y(s-v+1)), \quad t \in[-1, b]_{\mathbb{N}_{-1}} .
$$

We state three hypotheses that will be used below.

$\left(\mathrm{H}_{1}\right) f(t-v+1, x) \geq 0,(t, x) \in[v-1, b+v-1]_{\mathbb{N}_{v-1}} \times[0,+\infty)$;

$\left(\mathrm{H}_{2}\right) f(t-v+1, x)=h(t-v+1) g(x)$, where $h$ is a positive function, $g$ is a nonnegative function, and $\lim _{x \rightarrow 0^{+}} \frac{g(x)}{x}=0, \lim _{x \rightarrow \infty} \frac{g(x)}{x}=\infty$;

$\left(\mathrm{H}_{3}\right) f(t-v+1, x)=h(t-v+1) g(x)$, where $h$ is a positive function, $g$ is a nonnegative function, and $\lim _{x \rightarrow 0^{+}} \frac{g(x)}{x}=\infty, \lim _{x \rightarrow \infty} \frac{g(x)}{x}=0$.

Lemma 4 Assume condition $\left(\mathrm{H}_{1}\right)$ holds. Then $T y \in \mathcal{P}_{0}$ for all $y \in \mathcal{P}$. In particular, the operator $T$ leaves the cone $\mathcal{P}_{0}$ invariant. 
Proof For all $y \in \mathcal{P}$, by Theorem 7 and $\left(\mathrm{H}_{1}\right)$, we have $T y(t) \geq 0$ for all $t \in[-1, b]_{\mathbb{N}_{-1}}$. Further, it follows immediately from Theorem 7 (iii) that

$$
\min _{t \in\left[\frac{b}{4}, \frac{3 b}{4}\right]}(T y)(t) \geq \gamma \sum_{s=v-1}^{b+v-2} \max _{t \in[-1, b]_{\mathbb{N}-1}} G(t, s) f(s-v+1, y(s-v+1)) \geq \gamma\|T y\| .
$$

Therefore, $T y \in \mathcal{P}_{0}$.

Theorem 8 Assume that conditions $\left(\mathrm{H}_{1}\right)$ and $\left(\mathrm{H}_{2}\right)$ are satisfied. Then the FBVP (3.3) has at least one solution $y \neq 0 \in \mathcal{P}_{0}$.

Theorem 9 Assume that conditions $\left(\mathrm{H}_{1}\right)$ and $\left(\mathrm{H}_{3}\right)$ are satisfied. Then the FBVP (3.3) has at least one solution $y \neq 0 \in \mathcal{P}_{0}$.

The proofs of Theorem 8 and 9 are similar to Theorems 4.1 and 4.2 in [24] and are skipped.

\section{Competing interests}

The authors declare that they have no competing interests.

\section{Authors' contributions}

$\mathrm{CH}$ and $\mathrm{ZX}$ worked together in the derivation of the mathematical results. Both authors read and approved the final manuscript.

\section{Author details}

'School of Economics and International Trade, Zhejiang University of Finance and Economics, Hangzhou, Zhejiang 310018, P.R. China. ${ }^{2}$ Department of Mathematics, Yanbian University, Yanji, 133002, P.R. China.

\section{Acknowledgements}

The authors would like to thank the referee for invaluable comments and insightful suggestions. This work was supported by NSFC project (No. 11161049).

Received: 9 April 2015 Accepted: 25 August 2015 Published online: 17 September 2015

\section{References}

1. Atici, FM, Eloe, PW: A transform method in discrete fractional calculus. Int. J. Difference Equ. 2, 165-176 (2007)

2. Goodrich, CS: Solutions to a discrete right-focal fractional boundary value problem. Int. J. Difference Equ. 5, 195-216 (2010)

3. Atici, FM, Eloe, PW: Initial value problems in discrete fractional calculus. Proc. Am. Math. Soc. 137, 981-989 (2009)

4. Atici, FM, Sengül, S: Modeling with fractional difference equations. J. Math. Anal. Appl. 369, 1-9 (2010)

5. Bastos, NRO, Ferreira, RAC, Torres, DFM: Discrete-time fractional variational problems. Signal Process. 91, 513-524 (2011)

6. Goodrich, CS: Continuity of solutions to discrete fractional initial value problems. Comput. Math. Appl. 59, 3489-3499 (2010)

7. Miller, KS, Ross, B: Fractional difference calculus. In: Proceedings of the International Symposium on Univalent Functions, Fractional Calculus and Their Applications, Nihon University, Koriyama, Japan, May 1988. Ellis Horwood Ser. Math Appl., pp. 139-152. Horwood, Chichester (1989)

8. Ferreira, RAC: Existence and uniqueness of solution to some discrete fractional boundary value problems of order less then one. J. Differ. Equ. Appl. 19, $712-718$ (2013)

9. Dahal, R, Goodrich, CS: A monotonicity result for discrete fractional difference operators. Arch. Math. (Basel) 102 , 293-299 (2014)

10. Holm, M: Sum and difference compositions and applications in discrete fractional difference calculus. CUBO 13, 153-184 (2011)

11. Anderson, DR: Solutions to second-order three-point problems on time scales. J. Differ. Equ. Appl. 8, 673-688 (2002)

12. Atici, FM, Eloe, PW: Linear systems of fractional nabla difference equations. Rocky Mt. J. Math. 41, 353-370 (2011)

13. Dahal, R, Duncan, D, Goodrich, CS: Systems of semipositone discrete fractional boundary value problems. J. Differ. Equ. Appl. 20, 473-491 (2014)

14. Ferreira, RAC: Positive solutions for a class of boundary value problems with fractional $q$-differences. Comput. Math. Appl. 61, 367-373 (2011)

15. Ferreira, RAC: A discrete fractional Gronwall inequality. Proc. Am. Math. Soc. 140, 1605-1612 (2012)

16. Ferreira, RAC, Goodrich, CS: Positive solution for a discrete fractional periodic boundary value problem. Dyn. Contin. Discrete Impuls. Syst., Ser. A Math. Anal. 19, 545-557 (2012) 
17. Goodrich, CS: Existence of a positive solution to a system of discrete fractional boundary value problems. Appl. Math. Comput. 217, 4740-4753 (2011)

18. Goodrich, CS: On a discrete fractional three-point boundary value problem. J. Differ. Equ. Appl. 18, 397-415 (2012)

19. Goodrich, CS: On a fractional boundary value problem with fractional boundary conditions. Appl. Math. Lett. 25, 1101-1105 (2012)

20. Goodrich, CS: On a first-order semipositone discrete fractional boundary value problem. Arch. Math. (Basel) 99 509-518 (2012)

21. Goodrich, CS: On semipositone discrete fractional boundary value problems with nonlocal boundary conditions. J. Differ. Equ. Appl. 19, 1758-1780 (2013)

22. Bastos, NRO, Mozyrska, D, Torres, DFM: Fractional derivatives and integrals on time scales via the inverse generalized Laplace transform. Int. J. Math. Comput. 11, 1-9 (2011)

23. Ferreira, RAC, Torres, DFM: Fractional $h$-difference equations arising from the calculus of variations. Appl. Anal. Discrete Math. 5, 110-121 (2011)

24. Atici, FM, Eloe, PW: Two-point boundary value problems for finite fractional difference equations. J. Differ. Equ. Appl. $17,445-456$ (2011)

25. Goodrich, CS: On discrete sequential fractional boundary value problems. J. Math. Anal. Appl. 385, 111-124 (2012)

26. Atici, FM, Guseinov, G: On Green's functions and positive solutions for boundary value problems on time scales. J. Comput. Appl. Math. 141, 75-99 (2002)

27. Anderson, DR, Hoffacker, J: A stacked delta-nabla self-adjoint problem of even order. Math. Comput. Model. 38(5-6), 481-494 (2003)

28. DaCunha, JJ, Davis, JM, Singh, PK: Existence results for singular three point boundary value problems on time scales. J. Math. Anal. Appl. 295, 378-391 (2004)

29. Anderson, DR, Avery, RI: An even-order three-point boundary value problem on time scales. J. Math. Anal. Appl. 291, 514-525 (2004)

30. Guseinov, G: Self-adjoint boundary value problems on time scales and symmetric Green's functions. Turk. J. Math. 29(4), 365-380 (2005)

31. Wang, P, Wang, Y: Existence of positive solutions for second-order $m$-point boundary value problems on time scales. Acta Math. Appl. Sin. Engl. Ser. 22, 457-468 (2006)

32. Anderson, DR, Hoffacker, J: Existence of solutions for a cantilever beam problem. J. Math. Anal. Appl. 323, 958-973 (2006)

\section{Submit your manuscript to a SpringerOpen ${ }^{\ominus}$ journal and benefit from:}

- Convenient online submission

Rigorous peer review

- Immediate publication on acceptance

- Open access: articles freely available online

- High visibility within the field

- Retaining the copyright to your article 